\title{
Managing Vehicle Acceleration Properties by Programming Functions for Engine Torque Control
}

\author{
Ivan Dunđerski \\ University of Belgrade, School of Electrical Engineering and Computer Science Applied Studies, Serbia
}

A new vehicle acceleration properties control system has been developed to improve passenger comfort. Nonlinearity of acceleration influences sensitive passengers, who experience nausea while in a moving vehicle. This control system implements advanced engine torque management for control of the pulling force, which defines vehicle acceleration dynamics with regard to passenger comfort.

A theoretical model for the linear acceleration of the vehicle was developed. The function was defined to control acceleration intensity, and it determines conditions for acceleration properties to be linear. Programming code was written to manage engine charge according to acceleration function. A controller has been developed to be the executive device of the programming code, and to manage engine charge.

In the experiments, the variable acceleration is converted to constant with the choice of acceleration intensity value, as the driving parameter.

Keywords: vehicle acceleration function, mass jerk, engine torque programming control

\section{INTRODUCTION}

Vehicle motion dynamics is a highly developed field. Safety is steadily increasing, fuel consumption is being reduced, and drive comfort is improving.

In pursuing vehicle safety, many physical and mathematical models to control the behaviour of vehicle at variable speed, on straight and curved paths, on dry or slippery roads, have been developed. The stability and handling of the vehicle are related to the vector of the friction force between the wheel and the road [1]. The skidding of driving wheels during acceleration is prevented by traction control systems (TCS). Wheel locking while braking is prevented by the anti-lock braking system (ABS). Traction control with all-wheel drive is more complex than the control of two-wheel drive, because the information about the magnitude of traction cannot be obtained on the basis of differences in the rotation speed of the driving and non-driving wheels [2]. While moving through a curve, the lateral sliding of vehicle and vehicle spin around a vertical axis is controlled by an electronic stability program (ESP). By rule, the system integrates both TCS and ABS systems [3].

It is possible to control driving wheel skidding during vehicle acceleration via engine torque and braking the wheels. Control of torque eliminates the cause of skidding [4]. The feedback quickly changes the engine charge for engine torque to follow the nature of the skidding. The consequence is the unstable operation of the engine and the increased toxicity of exhaust gases [5]. Traction control by braking driving wheels eliminates that consequence by "cutting off" excess torque. The system comes into effect only after skidding occurs. Operation of the system creates noise and vibrations, and wears the friction pairs of the braking system. Most of the applied models have feedback, and they reduce engine torque while simultaneously braking driving wheels. Excess torque keeps the system in operation on the verge of skidding, and cutting off the excess torque is minimal.

A reduction of fuel consumption is achieved by reducing engine torque and delaying responses to the gas pedal [6]. The original program maps for the engine charge were "remapped". The driver is influenced by monitoring his driving style and receiving advice for vehicle movement, especially for the way of acceleration [7]. According to the classic laws of physics, the total increase (change) of vehicle kinetic energy does not depend on the way the vehicle accelerates. In fact, by reducing the torque, engine unsteadiness is reduced, because smaller torque yields smaller acceleration of the vehicle. With minor changes of speed, the engine consumes less fuel.

To summarise, engine torque is a common factor to all systems of controlling vehicle dynamics.

The driving comfort of passengers has, thus far, been improved by controlling vehicle oscillations on the road. Active suspension systems have been developed to reduce the oscillation amplitudes, extend the period of oscillations and to reduce the vehicle inclination in a curve. Such systems are complex and expensive, because they are based on programming management and energy-demanding devices, with strong amplification, acting on the suspension system. Optional three-level systems have also been developed. The driver selects the characteristics of oscillations, depending on the road and his wishes. The vehicle body height above the road and the intensity 
of oscillation damping are changed. Along with safety, comfort is improved by reducing vibrations and noise and by air-conditioning the passenger cabin [8].

Improving the driving comfort via vehicle dynamics is a relatively new technique. At first glance, it is difficult to connect driving comfort to the engine torque and find a way to achieve it.

The most comfortable drive is along a straight line is at a constant vehicle speed, when there are no inertia effects on passengers. In reality, such driving is possible only on a highway over a longer period of time.

The vehicle's velocity vector changes direction when turning, and intensity while decelerating or accelerating. Moving through the curves and decelerating (braking) is basically a question of vehicle dynamics safety, not comfort. The remaining area where driving comfort can be improved, according to the velocity vector, is in the accelerated motion of the vehicle along a straight line. In that sense, it is possible to control the intensity of the acceleration through its properties, which are the magnitude and rate of change.

The intensity of the acceleration determines the magnitude of inertia effect on the passengers.

Minimal intensity is defined by law of the minimal dynamic characteristic of the vehicle.

Change of acceleration intensity causes the vehicle to jerk, which affects the passengers. Variable acceleration creates Mass Jerk-Yank. In technical terms, mass jerk is well known. It is always taken into account when determining the motion dynamics of robot manipulation arms, tools for computer controlled machines $(\mathrm{CNC})$, crank mechanisms, cam couples, etc.

Jerk and mass jerk also occur during accelerated vehicle motion due to variable engine torque. When the engine speed increases, the torque rises, reaches maximum and then decreases. Vehicle transmission reflects variable engine torque into the variable pulling force of the vehicle. As the pulling force increases and decreases, the acceleration intensity changes along with it, producing jerk and mass jerk of a variable sign.

Jerk creates effects similar to the effect of a ship tilting on lateral waves, also with a variable sign. Sensitive passengers experience nausea, called "seasickness" [9]. In order to reduce the intensity and jerk direction change, passenger ships are equipped with gyroscopic masses. The gyroscope diverts the acting force so that the lateral oscillations of the ship around the longitudinal axis are transferred into longitudinal oscillations around the lateral axis.
Ship inertia around the lateral axis is significantly greater than inertia around the longitudinal axis, so the amplitude of the oscillation decreases while the period of the ship oscillations increases. In that way, the comfort of cruising is also improved for the passengers who are not sensitive to the seasickness.

In order to improve driving comfort during accelerated vehicle motion, the jerk should be eliminated. Nonlinear vehicle acceleration should be converted into a linear (constant) one. For this purpose, part of the vehicle pulling force, which remains available after overcoming moving resistance, should be constant along the vehicle acceleration path. Controlling the vehicle movement dynamics by controlling engine torque eliminates jerk and improves passenger comfort.

In order to control engine torque during vehicle acceleration, the engine torque curve family for acceleration is required. This family differs from the stationary state torque family. Part of the engine torque, which accelerates the vehicle, is reduced in relation to the stationary state due to the inertia of masses that move in a straight line (translate) and rotate, which are not present while the vehicle is moving at a constant speed. In addition to the influence of inertia, during accelerated vehicle motion, the engine works in an unsteady state, and the engine efficiency is decreased.

There are test benches at which the engine torque family is obtained for accelerated vehicle movement. Resistances to accelerated vehicle movement are simulated on the bench by means of programming management [10]. However, accurate results can be obtained only by recording the vehicle motion in real conditions. Therefore, the engine torque family in this research is obtained by recording acceleration data of the experimental vehicle.

For the model of the accelerated vehicle motion dynamics, university textbooks were used [11] and [12].

\section{PHYSICAL - MATHEMATICAL MODEL}

\subsection{Balance of Forces Equations and Resistance to Motion}

During accelerated vehicle movement on the road, vehicle pulling force $F_{\text {pull }}$ acts against road resistance $R$ and inertia resistance $R_{i n}$. The balance equation of vehicle pulling force with movement resistance during acceleration is expressed by a differential equation

$$
\begin{gathered}
{[11]:} \\
F_{p u l l}=K A v^{2}+G f \cos \alpha+G \sin \alpha \pm \frac{G}{g} \frac{d v}{d t} \pm J \frac{d v}{d t} \frac{1}{r_{d}^{2}}, \\
F_{\text {pull }}=R+R_{\text {in }} .
\end{gathered}
$$


Road resistances are often referred to as "outside resistance", because they act on the vehicle from "outside".

Pulling force $F_{\text {pull }}$ is produced by engine torque $T$, which is conducted through the transmission to the vehicle drive wheel [11]:

$$
F_{\text {pull }}=\eta_{t r} i \cdot \frac{T}{r_{d}} .
$$

Road resistances $R$ during the vehicle movement are:

$$
R=F_{D}+F_{r r}+F_{c l}, \quad R=\eta_{t r} i \cdot \frac{T_{R}}{r_{d}}
$$

Road resistance elements are:

$$
\begin{gathered}
F_{D}=0.5 \rho \cdot c_{x} \cdot A \cdot v^{2}, \\
F_{r r}=m g \cdot f \cdot \cos \alpha, \quad F_{c l}=m g \cdot \sin \alpha,
\end{gathered}
$$

where $F_{D}$ and $K=0.5 \rho c_{x}$ are given in [11]. Vehicle weight $G$ is substituted as $m g$.

Resistances $R$ of the road exist during constant speed and accelerated vehicle movement. A vehicle accelerates, overcoming inertial resistances $R_{\text {in }}$ by force $F$, which is part of the pulling force. The force $F$ is generated from a portion of engine torque $\Delta T$ that remains available after overcoming road resistance $R$ to movement.

$$
R_{i n}=m \frac{d v}{d t}+J \frac{d \omega}{d t}, \quad F=\eta_{t r} i \frac{\Delta T}{r_{d}}, \quad \Delta T=T-T_{R}
$$

Resistances $R_{i n}$ to acceleration exist only during accelerated movement of the vehicle. The first member $R_{\text {in }}$ in Eq. (5) represents the inertia of translating mechanical elements, and the second member represents the inertia of rotating mechanical elements.

Fig. 1 shows the physical model of the vehicle movement, while accelerating along the straight path (the often used "bicycle" model for $1 / 2$ of the vehicle).

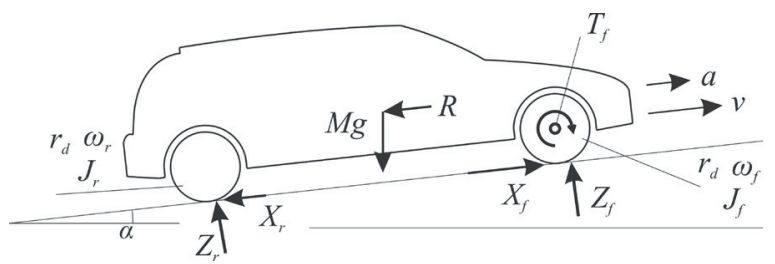

Fig. 1. Forces and torques acting on the vehicle during accelerated motion
With the path being horizontal, $\alpha=0$, the equation of balance of the forces and torques for vehicle on the horizontal road is:

$$
M \cdot a=X_{f}-X_{r}-R .
$$

Equations of balance of forces and torques of the front wheel and the equation of balance of forces and torques of the rear wheel are:

$$
T_{f}-X_{f} r_{d}=J_{f} \dot{\omega}_{f}, \quad X_{r} r_{d}=J_{r} \dot{\omega}_{r} .
$$

Summing equation (Eq. (7)) gives:

$$
T_{f}-\left(X_{f}-X_{r}\right) r_{d}=J_{f} \dot{\omega}_{f}+J_{r} \dot{\omega}_{r} .
$$

Eliminating the tangent reactions $\left(X_{f}-X_{r}\right)$ from Eq. (8), using Eq. (6), gives:

$$
M \cdot r_{d} \dot{\omega}_{f}+R=\frac{1}{r_{d}}\left[T_{f}-\left(J_{f} \dot{\omega}_{f}+J_{r} \dot{\omega}_{r}\right)\right], \quad a=r_{d} \dot{\omega}_{f} \cdot(9)
$$

In experiments, the angular speed of each wheel was measured via sensors for wheel turning, which showed that the angular speeds of the front and rear wheels were almost identical. Therefore, the mathematical model can be simplified:

$$
\dot{\omega}_{f}=\dot{\omega}_{r}=\dot{\omega} .
$$

Substituting Eq. (10) into Eq. (9) gives:

$$
\left(M r_{d} \dot{\omega}+R\right) r_{d}=T_{f}-J \dot{\omega}, \quad J=J_{f}+J_{r} .
$$

From Eq. (11), torque equation for the driving wheel of the vehicle is obtained:

$$
T_{f}-T_{f R}=\left(M r_{d}^{2}+J\right) \dot{\omega}, \quad T_{f R}=R \cdot r_{d} .
$$

\subsection{Engine Speed during Accelerated Motion}

Engine torque $T$ and road resistance torque $T_{R}$ are obtained from engine torque $T_{f}$ and resistance torque $T_{f R}$ acting on the driving wheel, using overall transmission gear ratio $i$. Acceleration $d n / d t$ of engine is obtained from the acceleration $d \omega / d t$ of the driving wheel using the overall transmission gear ratio $i$ and relation of angular speed to frequency $\omega=2 \pi n$ ( $n$ is an integer).

$$
T_{f}=i T, \quad T_{R}=i T_{f R}, \quad i \cdot \dot{\omega}=2 \pi \frac{d n}{d t} .
$$

Substituting the value from Eq. (13) to Eq. (12) gives: 


$$
i\left(T-T_{R}\right)=\left(M r_{d}^{2}+J\right) \frac{2 \pi}{i} \cdot \frac{d n}{d t} .
$$

From Eq. (14) arises the equation for engine speed rate of change $d n / d t$ during vehicle acceleration as a function of engine torque $T$ and road resistance torque $T_{R}$ on the engine:

$$
\frac{d n}{d t}=\frac{i^{2}}{2 \pi\left(M r_{d}^{2}+J\right)} \cdot\left(T-T_{R}\right) .
$$

In Eq. (15), the difference $\left(T-T_{R}\right)$ between engine torque and road resistance torque on the engine is torque $\Delta T$ that remains available to accelerate the vehicle after overcoming the road resistance $R$.

$$
\Delta T=T-T_{R}, \quad k=\frac{i^{2}}{2 \pi\left(M r_{d}^{2}+J\right)} .
$$

The constant $k$ determines the influence of overall transmission gear ratio $i$ and the inertia of masses $M$ that translate and masses $J$ that rotate during vehicle acceleration.

\subsection{Engine Torque Curve Family}

From Eq. (15), engine torque $T$, required to overcome the road resistance torque $T_{R}$ and the inertia $k^{-1} \cdot(d n / d t)$ during accelerated vehicle movement, is calculated:

$T=\frac{2 \pi\left(M \cdot r_{d}^{2}+J\right)}{i^{2}} \cdot \frac{d n}{d t}+T_{R}, \quad T=k^{-1} \cdot \frac{d n}{d t}+T_{R}$.

Eq. (17) is used to obtain a family of torque curves for accelerated vehicle moving. Engine speed $n$ during acceleration of the vehicle is taken from the speed sensor of the crankshaft. With engine speed $n$ in time $t$ of vehicle acceleration being known, the dependence $n=f(t)$ is also known, and it is used to calculate the rate of change $d n / d t$ in time $t$. Torque $T_{R}$ of resistance is calculated according to Eqs. (3) and (4).

Digital data on values of engine family torques $T$, in relation to flap angle $\theta$ (engine charge) and the engine speed $n$, are stored in the matrix (Eq. (18)), (lookup table for the torque family), from which they are read by the controlling program during vehicle acceleration.

Fig. 2 shows a graphical representation of the engine torque family. For this representation, the curve families are drawn "smoothly" through the points of the torque family from the matrix (Eq. (18)), by a special algorithm for data processing.
To define the gas pedal position and thereby the step of engine charge (load) per torque family, at an earlier stage, a mechanical spacer was used, working on the principle of the screw and nut. The electronic throttle control (ETC) pedal was physically limited to a certain height from vehicle floor.

At a later stage, a function for the controller was written to determine gas pedal position as a percentage of the deflection angle (\% DA) of ETC. The minimal step value is 1 as an integer. Data on the family are formed in steps of $2 \% \mathrm{DA}$ throughout the entire range, except for the two curves with a minimum charge, 25 and $28 \% \mathrm{DA}$, where the step is $3 \% \mathrm{DA}$ because of poor engine performance. The maximum physical position of the accelerator pedal position (full throttle) corresponds to $80 \%$ DA. This correspondence comes from the fact that the ETC deflection angle must be greater than the accelerator pedal position at full throttle. Otherwise, the ETC DA sensor could be damaged. Consequently, full throttle on Fig. 2 is given as $78 \% \mathrm{DA}$ and represents an "outside engine torque characteristic", which is the maximal torque that the engine can produce throughout the engine speed range.

The curves were recorded multiple times in order to verify them. The vehicle acceleration path is a straight line, the road is horizontal, and the surface is hard (asphalt) and dry.

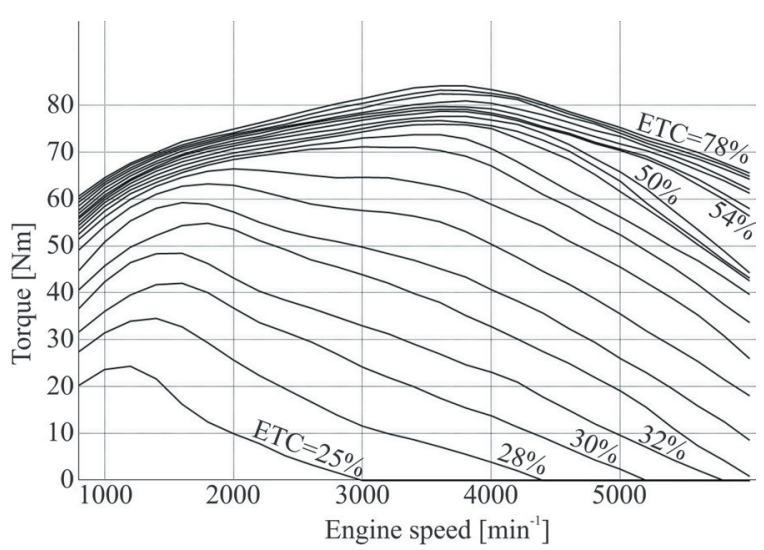

Fig. 2. Engine torque curves family

Conclusions from Fig. 2 about the shapes of curves are that above $50 \% \mathrm{DA}$, torque curves are similar in their shape/nature. Full engine performance is not changed for safety reasons, which are a priority. Naturally aspirated Otto engines, such as the one in the experimental vehicle, with an increase in engine speed, have an increase, a peak and a decrease in value of torque (also inflection points). 
Below 50\% DA, torque curves differ in their shape/nature. Torque maximums are shifted to lower engine speeds, and the curves have two maximums.

The increase in torque at lower engine speed allows moving the engine operation points from higher speeds to lower speed intervals. Engine operation with a smaller number of cycles saves fuel because the piston friction path is shortened, and the engine life is extended. The increase in torque (and power) at lower speeds is a feature of turbocharged engines, here achieved by remapping fuel maps of the engine control unit (ECU).

Conclusions from Fig. 2 about the density of curves are:

1. Above $50 \%$ DA, engine performances are maximal, because the fuel is not saved at the expense of vehicle safety. The curves' density is such that virtually all is "full throttle".

2. Below $50 \% \mathrm{DA}$, at which the engine performance differs, the curve density is lower. This allows more accurate control of engine charge via the gas pedal.

\subsection{Engine Torque and Acceleration Function}

Values of torques $T$ of the engine family torque curves in the matrix (look-up table) are defined by:

$$
\left(\begin{array}{ccc}
T_{11} & \ldots & T_{1 q} \\
\vdots & \ddots & \vdots \\
T_{p 1} & \cdots & T_{p q}
\end{array}\right),
$$

where $T_{i j}$ is torque of the engine, for engine speed $n_{i}$ and flap angle $\theta_{j}$ of engine aspiration system.

There is no explicit mathematical relation between engine torque and engine speed. The flap angle $\theta$, needed to obtain the desired torque $T$ at number $n$ of engine speed, is calculated with numerical methods, from the matrix Eq. (18).

$$
T=\theta(n) .
$$

The function $\theta(n)$ of acceleration in Eq. (19) determines the desired torque $T$ for a given engine speed $n$.

The choice of acceleration properties gives the acceleration function curve $\theta(n)$, which generally intersects the family curves $T(n ; \theta)$ of the engine torques. The way in which the acceleration function curve will intersect the engine torque curve family is determined by the chosen properties of the acceleration.
The programming model of the acceleration function $\theta(n)$ is stored in the controller module as an adaptive torque generator (ATG), shown in Fig. 3.

The programming model code is connected with the executable code of the controller. The executive code of the controller is stored in the controller module for flap angle calculation, called the throttle position calculator (TPC).

The ATG program module sends signals for torque values to the calculation module TPC $T_{A T G}$ (desired torque), in each controller cycle. The values of the desired torques are calculated according to acceleration function $\theta(n)$.

The TPC module calculates the flap angle $\theta_{A T G}$ on the basis of value of the $T_{A T G}$ torque, obtained from ATG and the current engine speed $n$. Signals carrying the value of the angle $\theta_{A T G}$ are relayed by the flap angle calculator to the ECU. In response to these signals, the ECU sets the flap to angle $\theta_{e}=\theta_{A T G}$ and thereby determines the charge (load) of the engine.

The controller is inserted between ETC and ECU, which were installed by vehicle manufacturer, and connected to the common "drive by wire" control system. At the start of acceleration, the driver determines desired final speed of the vehicle by using the gas pedal. The controller stores the driver's desire and controls acceleration until the desired vehicle speed is reached. Once the desired speed is reached, the controller returns control to the driver.

During acceleration the ECU "does not know" that it receives information about the value of the accelerator pedal position from the controller, not from the driver.

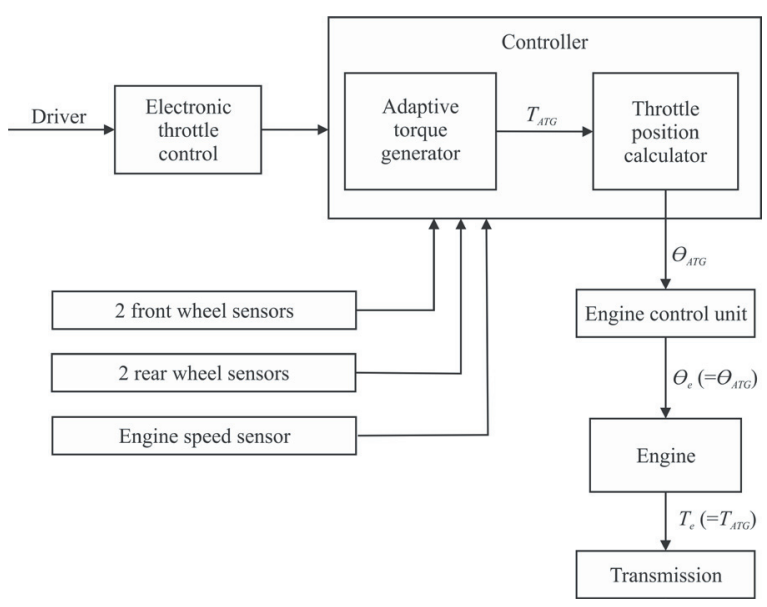

Fig. 3. Block diagram of Torque controller

The driver sets the desired ETC position; the ETC transmits the signal to the controller; the controller determines engine torque $T_{A T G}$ and its corresponding 
throttle valve angle $\theta_{A T G}$ and sends it to the engine ECU; the engine ECU sets the throttle valve angle $\theta_{e}$ to the calculated value $\theta_{A T G}$; the engine produces engine torque Te whose value is $T_{A T G}$, and delivers it to the transmission. The current engine speed $n$ is monitored and sent back to ATG controller.

In Fig. 4, the image of the controller is shown.



Fig. 4. Controller

Both the program model with its managing code and the controller with its executable code have been developed specifically for this research.

This research explores function of acceleration with property that the differences between engine torque $T$ and torque $T_{R}$ of resistance on the engine are constant. The vehicle accelerates without jerk with the desired intensity of acceleration. The constant difference of torques yields a constant difference $F=$ const part of force $F_{\text {pull }}$ that pulls the vehicle, which is available for vehicle acceleration after overcoming resistance $R$ to motion: $F=F_{\text {pull }}-R$.

\subsection{The Function of Torque Constant Differences}

Jerk $j$ is defined as the third derivative of the position vector with respect to time, which is the rate of change of the acceleration $a$.

For the acceleration $a$ along the $x$-axis, jerk $j$ is (scalar) [13]:

$$
j=\frac{d a}{d t}=\frac{d^{2} v}{d t^{2}}=\frac{d^{3} x}{d t^{3}}, \quad j=\dot{a}=\ddot{v}=\dddot{x} .
$$

Mass jerk $Y$ (Yank), [13] which is caused by jerk $j$ during variable acceleration of the mass $m$ is by definition:

$$
\begin{gathered}
Y=m \cdot j=m \frac{d a}{d t}=\frac{d}{d t}(m a), \\
F=m \cdot a \Rightarrow Y=\frac{d}{d t}(F)=\frac{d F}{d t} .
\end{gathered}
$$

Mass jerk $Y$ causes non-constant force $F$, which accelerates mass $m$, i.e. vehicle.

Mass jerk $Y$ can be obtained starting with second Newton's law $F=m a$ and applying derivative by time: $(d / d t) F=(d / d t) m a=m(d a / d t), Y=d F / d t=m j$.

Jerk $j$ on a vehicle arises from changing the intensity $d^{2} \omega / d t^{2}$ of angular acceleration of the driving wheels. The angular speed $\omega$ is related to the speed $v$ of vehicle movement on a straight path, with expression $v=r_{d} \omega$, where $r_{d}$ is the dynamic radius of the wheel. Using this in Eq. (20) gives the jerk of the vehicle:

$$
j=\frac{d^{2} v}{d t^{2}}=\frac{d^{2}\left(r_{d} \omega\right)}{d t^{2}}=r_{d} \frac{d^{2} \omega}{d t^{2}}=r_{d} \ddot{\omega}, \quad v=r_{d} \omega .
$$

Change of intensity $d^{2} \omega / d t^{2}$ of the angular acceleration of the driving wheels occurs by changing the intensity $d^{2} n / d t^{2}$ of the angular acceleration of the crankshaft.

Jerk $j_{e}$ of the angular acceleration on the engine crankshaft is obtained through the overall transmission gear ratio $i$ and angular speed to circular frequency relation $\omega=2 \pi n$ for the whole number $n$ of revolutions.

$$
j_{e}=\eta_{t r} \cdot i \cdot j=2 \pi r_{d} \eta_{t r} i \cdot \ddot{n}, \quad \omega=2 \pi n .
$$

Mass jerk $Y_{F}$ on vehicle depends on the intensity $d F / d n$ of change of the force vector $F$, Fig. 5, which accelerates the vehicle along the $x$-axis and causes a jerk $j_{e}$ on the engine. According to Eq. (21) it follows:

$$
Y_{F}=\frac{d F}{d t}=\frac{d F}{d n} \cdot \frac{d n}{d t}, \quad \frac{d F}{d n}=\frac{Y_{F}}{\dot{n}} .
$$

In Eq. (24), the change $d n / d t$ of engine speed is determined by Eq. (15). The force $F$ which accelerates the vehicle is part of the force $F_{\text {pull }}$ (Eq. (2)) of pulling, which remains available for acceleration of the vehicle after overcoming resistance $R$ to motion (Eq. (3)):

$$
F=F_{\text {pull }}-R \text {. }
$$

The force $F$ accelerates the vehicle overcoming inertia while accelerating (Eq. (5)).

Fig. 5a shows curves of force $F_{\text {pull }}=f(n)$ of the vehicle pulling in dependence to engine speed $\mathrm{n}$ according to Eq. (2) and forces $R=g(n)$ of resistance to movement of the vehicle Eq. (3), where the engine speed $n=\omega i / 2 \pi$ is expressed by the angular speed $\omega$ of the wheel, the overall transmission gear ratio $i$ and the relation of the angular speed to the circular frequency.

In Fig. 5b, vertical segments $F_{q}$ represent the difference $F_{\text {pull }}-R$ between the vehicle pulling force 
and movement resistance force. These segments of pulling force remain available for vehicle acceleration, after overcoming resistances $R=g(n)$, and they represent values of force curve $F=h(n)$. Values $F_{q}$ in the top diagram are equal to values $F_{q}$ in the bottom diagram for each arbitrary $q$.

In the theoretical diagrams in Fig. 5, value $F_{1}$ corresponds to engine speed $n_{1}=n_{\min }$ which is the minimal engine speed required for the engine to operate in practice. Depending on the engine's construction, $n_{\min }$ varies from $(800 / 60)$ to $(1100 / 60)$ $\mathrm{s}^{-1}$.

The intersection of functions $f(n)$ and $g(n)$ corresponds to the maximal engine speed achievable for given external resistances. For that engine speed value, the engine does not have the pulling force needed to accelerate the vehicle further.

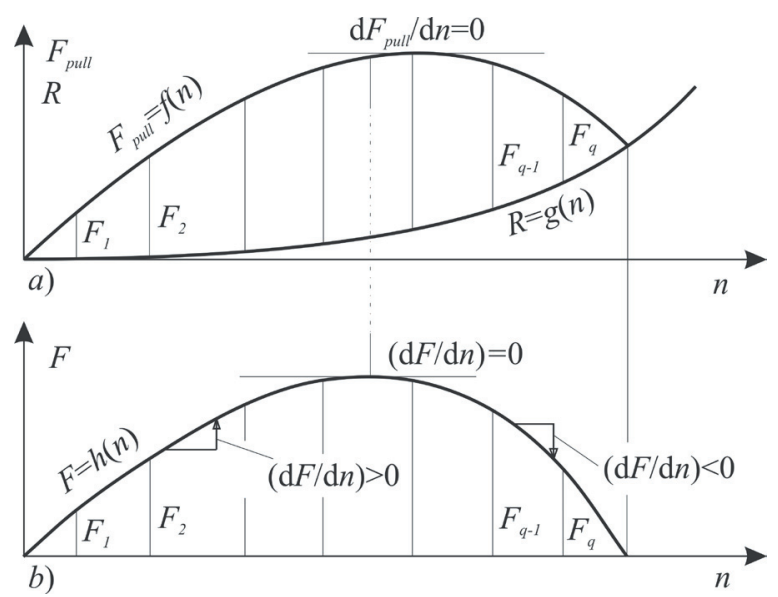

Fig. 5. a) Force Fpull $=f(n)$ of pulling and resistance $R=g(n)$ force; and $b)$ force $F=h(n)$ which accelerates the vehicle

Fig. $5 \mathrm{~b}$ shows that as the engine speed $n$ increases, the force $F$, which accelerates the vehicle at first rises; $d F / d n>0$, reaches maximum, $d F / d n=0$, and then falls, $d F / d n<0$.

Mass jerk, $Y_{F}$ created by changing intensity of force $F$, which accelerates the vehicle, acts in the direction of movement.

The condition for mass jerk elimination is obtained from Eq. (23), by putting that $j_{e}=0$ :

$$
\begin{aligned}
& j_{e}=2 \pi r_{d} \eta_{t r} i \cdot \ddot{n}=0, \\
& \frac{d \dot{n}}{d t}=0, \quad \dot{n}=\text { const } .
\end{aligned}
$$

From Eq. (26), it follows that there is no mass jerk when the change of angular speed during vehicle acceleration is constant, $d n / d t=$ const. According to Eqs. (16), (17) and (26), it follows:

$$
\begin{gathered}
\Delta T=T-T_{R}=\frac{2 \pi\left(M \cdot r_{d}^{2}+J\right)}{i^{2}} \cdot \frac{d n}{d t}, \\
\Delta T=k^{-1} \cdot \dot{n}=\text { const. }
\end{gathered}
$$

where $k$ is given in Eq. (16).

From Eq. (27), it follows for engine torque $T$ of:

$$
T=T_{R}+\Delta T, \quad \dot{n}=\text { const } .
$$

Torque $T$ is guided along acceleration function $\theta_{\Delta T}(n)$, so according to Eq. (28) the acceleration function is:

$$
\begin{array}{ll}
T=\theta_{\Delta T}(n), & \theta_{\Delta T}(n)=T_{R}(n)+k^{-1} \cdot \dot{n}, \\
& \dot{n}=\text { const } .
\end{array}
$$

When the condition $d n / d t=$ const is fulfilled, the vehicle accelerates by function $\theta_{\Delta T}(n)$ (Eq. (29)), with the property that acceleration is without jerk.

Eq. (29) represents acceleration function $\theta_{\Delta T}(n)$, which has the property that during vehicle acceleration, the difference $\Delta T$ between engine torque $T$ and resistance to motion torque $T_{R}$ on the engine is constant.

The value of torque difference $\Delta T$ that accelerates the vehicle in Eq. (27) is a variable arbitrary parameter that defines the intensity of the acceleration of the vehicle. Theoretically, to drive with the best comfort, the minimal value of the arbitrary parameter should be taken, because when $\Delta T \rightarrow 0$ acceleration is minimal, and the impact of inertia is minimal. In practice, the parameter value should satisfy legal limitations for the dynamic vehicle characteristic needed for safety during acceleration. Higher values of the chosen parameter increase vehicle acceleration, resulting in a greater influence of inertia on passengers, which decreases the comfort of driving.

Substituting into Eq. (2) that $F_{\text {pull }}=F_{\theta \Delta T}(n)$ and that $T=\theta_{\Delta T}(n)$ it follows:

$$
F_{\theta_{\Delta T}}(n)=\eta_{t r} i_{t r} \frac{\theta_{\Delta T}(n)}{r_{d}}
$$

The graphical representation of curve $F \theta_{\Delta T}(n)$ of vehicle pulling force, which corresponds to the function $\theta_{\Delta T}(n)$ acceleration without jerk, is given in Fig. 6a, and the graphical representation of curve $F=$ $h(n)$ force that accelerates the vehicle without jerk is given in Fig. 6b.

Each point of the function curve $F \theta_{\Delta T}(n)$ is located at the same distance $F_{q}=$ const along the ordinate, above the curve of function $R=g(n)$ (Fig. 6a). This 
distance represents intensity of part of pulling force, that accelerates the vehicle, (Fig. 6b). Therefore, the mass jerk $Y_{F}$ is eliminated along the largest part of the acceleration path. At both the start and the end of the acceleration, there is a short-term jerk, and variable force $F$ along with it, which accelerates the vehicle, because it is not possible to transit from constant speed movement to accelerated movement without jerk (similar to the movement of a numerical machine tool). However, the swinging effect is avoided.

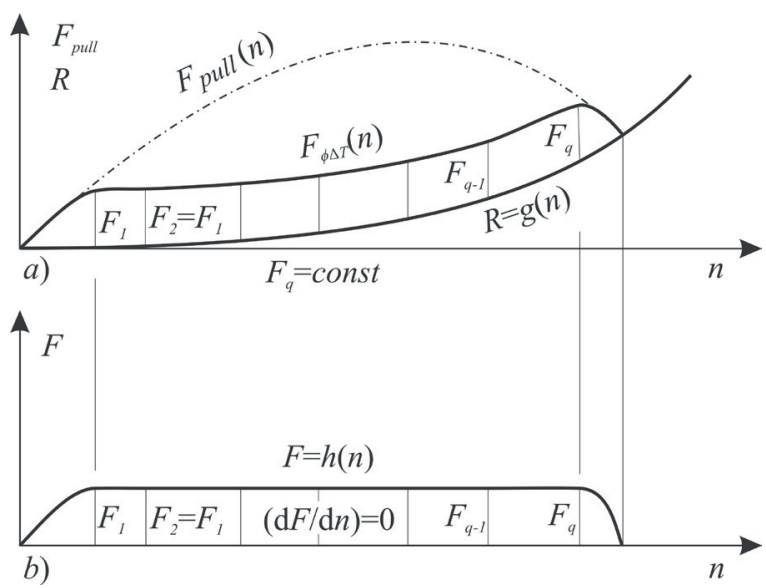

Fig. 6. Function $\mathrm{F}_{\Delta T}(\mathrm{n})$ of acceleration with constant difference $F_{q}=$ const in relation to resistance a) $R=g(n)$ of the road and $b$ ) force $F=h(n)$ which accelerate vehicle

When braking, the vehicle braking force is constant on the largest part of the path [14] and [8] so the diagram of braking force is very similar to the acceleration diagram in Fig. 6b, along with the negative sign (deceleration of the vehicle). This fact implies that the nonlinear acceleration of the vehicle is the primary cause for the effect of swinging during the drive.

\section{EXPERIMENTS}

\subsection{Computer Simulation}

In the early stage of research, the computer used Lyderman's formula [11] and [12], which gives an explicit mathematical relation of engine power to engine speed. Lyderman's formula for power was previously transformed into the formula for the torque.

At a later stage of research, the computer used a real torque family, obtained by recording on the vehicle and applying the Eq. (17).

Fig. 7 shows a computer simulation of acceleration with a constant difference $\Delta T=$ const of engine torque, above the curve $T_{R}(n)$ of resistance torque of the road, over a real family of engine torque curves of the experimental vehicle.

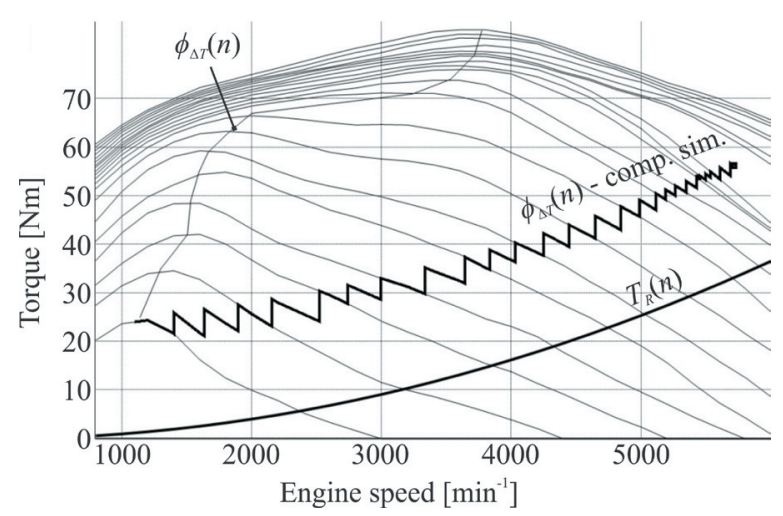

Fig. 7. Curve of constant difference of torques, computer

The curve $\theta_{\Delta T}(n)$ comp. sim. of engine torque lies above the curve $T_{R}(n)$ of the function of resistance torque of the road. Vertical segments between the two curves are equal by value across the entire range of engine speeds. The two curves correspond to the force $F$ that accelerates the vehicle, according to Eqs. (29) and (30).

Curve $\theta_{\max }(n)$ in Fig. 7 connects maximal torques of partial engine charges, illustrating the possibility of programming other acceleration functions.

The characteristic "jagged" shape of $\theta_{\Delta T}(n)$ comp. sim. originates from the discretization of the ETC flap angle. The vertical segments of the curve represent the discreet change of the $\Delta \theta_{e}$ angle, through which the engine charge (load) is changed. Sloped segments between vertical segments are for the same valve angle, $\theta_{e}=$ const. Across sloped segments, engine speed $n$ is changing along with engine torque, with the same flap angle (load).

The value of discrete change of the flap angle $\Delta \theta_{e}$ programming step is an arbitrary parameter, defined by the programming model. The minimal value of the arbitrary parameter for the vehicle is limited to the minimal step value of the mechatronic system that controls the flap angle.

\subsection{Vehicle}

Experiments were conducted on the VW Polo 1.2 laboratory vehicle with Simos N9.1 ECU Except for the installed controller, the vehicle is completely serial. 


\subsubsection{Experimental Setup}

The values of the physical parameters used in Eq. (4) related to vehicle and road during experiments are: $\rho=1.226 \mathrm{~kg} / \mathrm{m}^{3}, c_{x}=0.30, A=1.75 \mathrm{~m}^{2}, m=1200$ $\mathrm{kg}$ (vehicle mass with two passengers), $g=9.81 \mathrm{~m} / \mathrm{s}^{2}$, $f=0.018, \alpha=0$.

For practical calculations, the programming model uses a modified formula for $k$, given in Eq. (16):

$$
k=\frac{i^{2}}{2 \pi \cdot r_{d}^{2} \cdot \delta M}, \quad \delta M=M+\frac{J}{r_{d}^{2}},
$$

where coefficient $\delta$ is calculated according to $\delta=1+\delta_{1} \cdot\left(i_{g}\right)^{2}+\delta_{2}, \delta_{1}=0.040, \delta_{2}=0.035$ are adopted as mean values from [11]. The estimation of the coefficient $\delta$ is applied because of difficulties noted to determine $\delta$ by theoretical formula [11]. The value of coefficient $\delta=1+0.04 \cdot 1.392+0.035=1.11$. The value $\delta M=1.11 \cdot 600=666 \mathrm{~kg}$. The value $J$ itself is not needed for calculation, but it can be obtained as $J=\left(\delta_{1} \cdot\left(i_{g}\right)^{2}+\delta_{2}\right) M\left(r_{d}\right)^{2}=(0.04 \cdot 1.392+0.035) \cdot 600 \cdot$ $\cdot 0.2832=5.39 \mathrm{kgm}^{2}$. The $J$ value is small in respect to $M$, and thus has no significant influence to coefficient $k$ in practice.

Coefficient $k$ influences the magnitude of torque $T$ (Eq. (17)) but does not affect the nature of torque $T$ curve.

Values of physical parameters $i, M, r_{d}$ used in Eq. (31) related to vehicle during experiments are: $M=1 / 2 m, i=\omega_{e} / \omega_{f}$, and $i_{g}=i / i_{0}=i / 4.19$.

The programming model calculates overall transmission ratio $i$ for any transmission gear, using data from sensors for engine speed $\omega_{e}$ and front wheels' angular speed $\omega_{f}$. This simplifies the calculation algorithm by eliminating the need for additional input related to the current transmission gear and makes the programming model portable to any contemporary vehicle. In addition, no gearbox sensors are needed to inform the controller which gear is currently coupled.

Value of $r_{d}$ was calculated once according to Eq (32):

$$
r_{d}=\frac{v}{\omega_{f}}=\frac{i \cdot v}{\omega_{e}}=\frac{i \cdot v}{2 \pi n}
$$

where $v=41 \mathrm{~km} / \mathrm{h}=11.39 \mathrm{~m} / \mathrm{s}$ measured using navigation device (GPS), $n=2240 \mathrm{rpm}=37.33 \mathrm{~s}^{-1}$ measured using Bosch KTS 640 scanner, and $i=$ $i_{0}$ to $i_{3}=4.19$ to $1.39=5.82$ adopted from vehicle manufacturer specification. According to Eq. (32) and given values, $r_{d}$ is $0.283 \mathrm{~m}$.

\subsubsection{Experimental Results}

Fig. 9 shows the curve $\theta_{\Delta T}(n)$ of the acceleration function, obtained experimentally by recording on the vehicle. Aperiodic oscillations in the curve are due to the noise accompanying the recording, since the flap changes its angle in discrete steps. Between two changes, the flap is standing still.

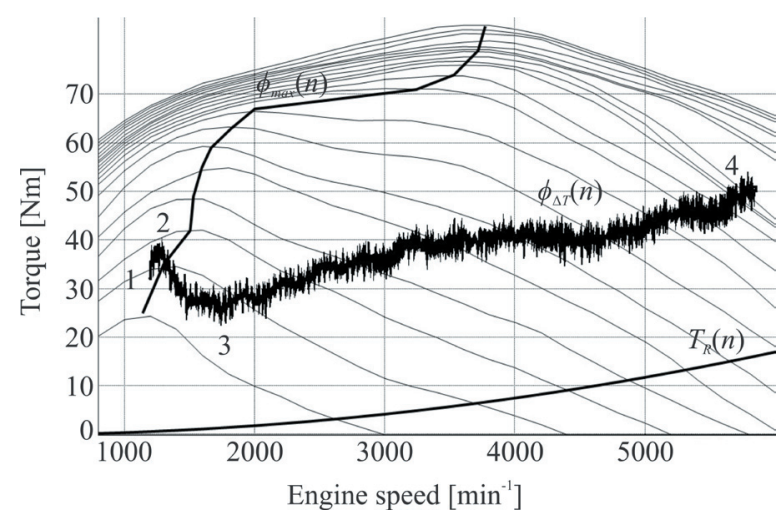

Fig. 8. Curve of constant torque difference, vehicle

The value of the optional parameter $\Delta T=$ const, (Eq. (27)) is available for the driver to set, by pressing the gas pedal. The curve in Fig. 9 was recorded with a value of $60 \% \mathrm{DA}$. Optional parameter $\Delta T$ corresponds to the force $F_{\theta \Delta T}(n)$, which accelerates the vehicle (Eq. (30)), by the function $\theta \Delta T(n)$ of acceleration (Eq. (29)). At the beginning of the acceleration path, the vehicle is moving at low speed in the third gear of the transmission. In this gear, the vehicle has a top speed over $100 \mathrm{~km} / \mathrm{h}$, at which the influence of air resistance is visible in the diagram of the experimentation results. In the first transmission gear, the vehicle's top speed is too low to produce air resistance sufficiently significant to be visible on the diagram.

At the starting moment, Point 1, on Fig. 8, the gas pedal is rapidly pressed and held down until the end of the acceleration, Point 4.

At Point 1, the starting of the acceleration curve, engine speed is $\sim 1200$ revolutions per minute (RPM), and the torque value is $\sim 33 \mathrm{Nm}$. With the pressing of the gas pedal, the engine charge (load) rises and a small increase of torque to $\sim 38 \mathrm{Nm}$ follows, along with a small increase of engine speed to $\sim 1300 \mathrm{rpm}$, point 2.

In Point 2, the control begins. The controller modifies current movement mode of the vehicle 
to achieve the desired movement according to acceleration function $\theta_{\Delta T}(n)$. The convergence of the actual torque curve to the desired torque curve, defined by acceleration function, takes place gradually during the transitional period between Points 2 and 3 . The controller calculates engine torque $T$ from the current engine speed $n$, flap angle $\theta$ and resistance torque $T_{R}$ of the road, depending on current vehicle speed. In each cycle, the desired torque $T_{A T G}$ is calculated so that its value is between engine torque $T$ and the value of acceleration function $\theta_{\Delta T}(n)$. In that way, the actual acceleration curve approaches the theoretical one $\theta_{\Delta T}(n)$, as engine speed $n$ increases in the interval between Point 2 and 3 (Fig. 8). This procedure avoids the jerk that would arise by rapid fall of engine torque $T$ to the value of the function $\theta_{\Delta T}(n)$. Rapid and complete gas seizure would cause the engine to go from pull to brake, reversing the pulling force direction, which would generate jerk.

From Point 3 to 4, the difference between torque $T$ and torque value of acceleration function $\theta_{\Delta T}(n)$ are small enough that desired torque $T_{A T G}$ matches the value of torque by function $\theta_{\Delta T}(n)$. In this way, the acceleration takes place by $\theta_{\Delta T}(n)$ a function of acceleration, with a $\Delta T(n)=$ const, according to Eqs. (27) and (29).

At Point 4, the vehicle acceleration ends by function $\theta_{\Delta T}(n)$ of acceleration (electronically limited maximum engine speed is reached with a sharp power drop).

From Fig. 8, it is clear that during vehicle acceleration, between Points 3 and 4, the vertical distances between the curves $\theta_{\Delta T}(n)$ and $T_{R}(n) n$ are practically the same. These distances represent the intensity of part of the engine torque $\Delta T=$ const that accelerates the vehicle, or remains available for increasing the vehicle speed after overcoming road resistance torque.

Control of torque between Points 3 and 4 is achieved by the programming model of the controller, and the influence of the driver on the vehicle movement is excluded.

\section{CONCLUSIONS}

\subsection{Introduced Concept}

This paper shows the concept for excluding mass jerk that affects vehicle during acceleration, which improves passenger driving comfort.

A new system for engine torque control has been developed. The system eliminates mass jerk on the largest portion of the vehicle acceleration path by maintaining constant vehicle acceleration intensity.

This principle can be applied to model and control other acceleration properties, such as engine operation efficiency, fuel consumption or driving wheel slip.

\subsection{Methodology Applied}

The physical and mathematical model is introduced to determine forces and torques acting on the vehicle during acceleration. According to the model, the pulling force is the sum of two parts. The first part overcomes resistance to vehicle motion on the road, while second part overcomes the inertia of rotating and translating masses.

Pulling force is maintained so that its part that overcomes inertia is of constant intensity. In this manner, vehicle mass jerk is eliminated during acceleration.

The acceleration function, programming model, and the controller were developed in order to control pulling force. The programming model instructs the controller to set the engine charge in respect to acceleration function. Managing the charge is discrete and iterative.

The torque curve family for the experimental vehicle was recorded in actual vehicle acceleration conditions on the road. The data regarding the torque family is arranged in matrix (lookup table). The acceleration function evaluates throttle angle that produces the desired torque in real time from the matrix for current engine speed.

Acceleration control is automatic. The driver sets the intensity of the acceleration by pressing the accelerator pedal only at the start of acceleration.

\subsection{Results of Research}

In the first phase of research, a special programming module was written to simulate vehicle acceleration. The module requires input for vehicle mass, current gear ratio, starting engine speed and desired acceleration intensity. The main focus of output is a torque curve, showing engine states through time. Other various data for current simulation state are shown, such as current power, vehicle speed, elapsed time, distance travelled, etc.

In the second phase of the research, the experiments were conducted while driving the vehicle on the road. During vehicle acceleration, the engine speed in time was recorded.

Analysis of data obtained by both simulation and experimentation on the vehicle shows good results for 
the control of pulling force intensity. On the output diagram, the engine torque curve is located above road resistance torque curve. The difference of torque values for these two curves is practically the same at any given engine speed. Therefore, the part of pulling force that accelerates vehicle is considered to be constant, eliminating mass jerk during acceleration.

\section{NOMENCLATURE}

$A$
$a$
$c_{x}$
$f$
$G$
$g$
$F$
$F_{c l}$
$F_{D}$
$F_{r r}$
$F_{p u}$
$i$
$i_{0}$
$i_{g}$
$J$
$J_{f}$

a vehicle acceleration $[\mathrm{m} / \mathrm{s} 2]$

$c_{x} \quad$ air drag coefficient [-]

$f \quad$ rolling resistance coefficient [-]

$G \quad$ vehicle weight [N]

$g$ gravity acceleration $\left[\mathrm{m} / \mathrm{s}^{2}\right]$

$F \quad$ vehicle acceleration force $[\mathrm{N}]$

$F_{c l} \quad$ climbing resistance force [N]

$F_{D} \quad$ air resistance (drag) force [N]

$F_{r r} \quad$ rolling resistance force [N]

$F_{\text {pull }} \quad$ vehicle pulling force [N]

$i \quad$ overall transmission gear ratio [-]

$i_{0} \quad$ main transmission gear ratio [-]

$i_{g} \quad$ gth gear transmission ratio [-]

$J \quad$ moment inertia of rotating masses $\left[\mathrm{kgm}^{2}\right]$

$J_{f} \quad$ moment inertia of rotating masses of front wheel, transmission elements, engine and clutch $\left[\mathrm{kgm}^{2}\right]$

$J_{r} \quad$ moment inertia of rear wheel $\left[\mathrm{kgm}^{2}\right]$

$j \quad$ jerk $\left[\mathrm{m} / \mathrm{s}^{3}\right]$

$M \quad$ physical model mass [kg]

$m \quad$ vehicle mass [kg]

$n \quad$ engine speed $\left[\mathrm{s}^{-1}\right]$

$R \quad$ road resistances [N]

$R_{\text {in }} \quad$ inertia resistances [N]

$r_{d} \quad$ dynamic radius of the driving wheel [m]

$T \quad$ engine torque $[\mathrm{Nm}]$

$T_{f} \quad$ engine torque transmitted to front driving wheel [Nm]

$T_{f R} \quad$ road resistance acting on front wheel as torque $[\mathrm{Nm}]$

$T_{R} \quad$ road resistance acting on engine as torque [Nm]

$v \quad$ vehicle speed $[\mathrm{m} / \mathrm{s}]$

$X_{f} \quad$ road tangent reaction to front wheel [N]

$X_{r} \quad$ road tangent reaction to rear wheel [N]

$Y \quad$ mass jerk $\left[\mathrm{kgm} / \mathrm{s}^{3}\right]$

$Y_{F} \quad$ vehicle mass jerk $\left[\mathrm{kgm} / \mathrm{s}^{3}\right]$

$Z_{f} \quad$ road normal reaction to front wheel [N]

$Z_{r} \quad$ road normal reaction to rear wheel [N]

$\alpha \quad$ road inclination angle $\left[{ }^{\circ}\right]$

$\delta \quad$ inertia influence coefficient of rotating masses [-] $\delta_{1} \quad$ inertia influence coefficient of rotating masses of engine and clutch [-]

$\delta_{2}$ inertia influence coefficient of rotating masses of drivetrain (wheels) [-]

$\eta_{t r} \quad$ transmission efficiency coefficient [-]

$\rho$ air density $\left[\mathrm{kg} / \mathrm{m}^{3}\right]$

$\omega$ angular speed of wheels [ $\mathrm{rad} / \mathrm{s}$ ]

$\omega_{f}$ angular speed of front wheels [ $\mathrm{rad} / \mathrm{s}$ ]

$\omega_{r}$ angular speed of rear wheels $[\mathrm{rad} / \mathrm{s}]$

\section{ABBREVIATIONS}

DA deflection angle of ETC

ECU engine control unit

ETC electronic throttle control

RPM revolutions per minute

TPC throttle position calculator

\section{REFERENCES}

[1] Canudas, C., Tsiotras, P., Velenis, E., Basset, M., Gissinger, G. (2003). Dynamic friction models for road/tire longitudinal interaction. Vehicle System Dynamics, vol. 39, no. 3, p. 189-226, DOI:10.1076/ vesd.39.3.189.14152.

[2] Deur, J., Pavkovic, D., Burgio, G., Hrovat, D. (2011). A model-based traction control strategy non-reliant on wheel slip information. Vehicle System Dynamics, vol. 49, no. 8, p. 1245-1265, DOI:10.1080/00423114.2010. 511675.

[3] Masuno, K., Nitta, R., Inoue, K., Ichikaw, K., Hiwatashi, Y. (2000). Development of a new all-wheel drive control system. Proceedings of 2000 FISITA World Automotive Congress, Seoul, Technical paper. no. F2000G347.

[4] Jae-Book, S., Byong-Cheol, K., Dong-Chul, S. (1999). Development of TCS slip control logic based on engine throttle control. KSME International Journal of Mechanical Science and Technology, vol. 13, no. 1, p. 74-81, DOI:10.1007/BF02946125.

[5] Shin, M., Han, J., Youn, J., Sunwoo, M. (2006). Design of a network-based traction control system using a formalized design procedure. Proceedings of Yokohama FISITA World Automotive Congress, Yokohama, Technical paper no. F2006SC22.

[6] Fuji Heavy Industries Ltd. (2007). Subaru intelligent drive (SI drive), from http://drive2.subaru.com/ Summer07_whatmakes.htm, accessed on 2013-02-06.

[7] Ford motor company newsroom (2011). All-new ford focus features ecomode to help drivers perfect ecodriving techniques, from http://media.ford.com/article display.cfm?article_id $=33965$, accessed on 2013-0208.

[8] Kost, F., Post, W. (eds.) (2006). Safety, Comfort and Convenience Systems. Robert Bosch GmbH, Cambridge. 
[9] Wikipedia. (2014). Motion Sickness, from http:// en.wikipedia.org/wiki/Motion_sickness, accessed on 2014-02-19.

[10] Joly, P., Duboc, S. (2000). Common rail mapping on power train test bench. Proceedings of Seoul 2000 FISITA World Automotive Congress, Seoul, Paper no. F2000A054.

[11] Janković, D., Ivanović, G., Todorović, J., Rakicević, B. (2001). The Theory of the Movement of Motor Vehicles.
Faculty of Mechanical Engineering, Belgrade. (in Serbian)

[12] Simić, D. (1977). Motor Vehicles. Science book, Belgrade. (in Serbian)

[13] Wikipedia. (2014). Jerk physics, from http:// en.wikipedia.org/wiki/Jerk_(physics), accessed on 2014-02-19.

[14] Todorović, B. (1988). Braking of Motor Vehicles. Department of textbooks and teaching aids, Belgrade. (in Serbian) 